\title{
STRATIFICATIONS SOCIALES EN AFRIQUE ANCIENNE: PERFORMANCES ET STAGNATIONS
}

Benjamin Diouf ${ }^{1}$

\begin{abstract}
Résumé
Les sociétés africaines ont eu des organisations qui ont évolué et se sont maintenues en dépit du temps qui nous sépare de l'Antiquité. Parmi celles-ci, nous avons les castes qui sont, pour certains, une absurdité qu'il faut abandonner sans chercher à les comprendre. Pourtant, celles-ci peuvent nous éclairer sur le fonctionnement de notre société et son évolution. Les castes sont une stratification sociale établie sur la base des activités professionnelles, des métiers. C'est en Egypte ancienne qu'elles vont se professionnaliser et s'hériter. Au fil du temps, la religion et la loi vont intervenir dans le système des castes pour y introduire l'endogamie, la hiérarchie et le mépris tels que nous l'observons, aujourd'hui, chez certaines ethnies africaines.
\end{abstract}

\section{Mots-clés}

Castes; classes sociales; stratifications sociales; organisations sociales; hiérarchie; endogamie; Egypte ancienne; fondement socio-politique; religieux; juridique.

\footnotetext{
${ }^{1}$ Assistant titulaire - Université Cheikh Anta Diop de Dakar, Dakar, Senegal. E-mail: benjdiouf067@yahoo.fr
}

Heródoto, Unifesp, Guarulhos, v.4, n.1 - 2019.1. p. 247-263

DOI: $10.34024 /$ herodoto.2019.v4.10121 


\begin{abstract}
African societies have had organizations that have evolved and remained steady despite the period of time that separates us from antiquity. Among these, we have the castes which are, for some, an absurdity that must be abandoned without trying to understand them. Yet, these can enlighten us as to the functioning of our society and its evolution. Castes are a social stratification established on the basis of professional activities and trades. It is in ancient Egypt that they will become professional and inherit. Over time, religion and law will intervene in the caste system to introduce endogamy, hierarchy and contempt as we can see it today, in some African ethnic groups.
\end{abstract}

\title{
Keywords
}

Castes, social classes, social stratification, social organizations, hierarchy, endogamy, ancient Egypt, socio-political, religious, legal foundation. 
Stratifications sociales en Afrique ancienne: performances et stagnations.

\section{Introduction}

Les sociétés africaines, à l'instar de toutes les autres, ont eu des organisations qui ont évolué avec le temps et ont réussi à se maintenir en dépit du temps qui nous sépare de l'Antiquité. En effet, certaines croyances et relations sociales peuvent être rapprochées de celles qui étaient jadis entretenues en Egypte, en Libye ou en Ethiopie anciennes. Celles-ci continuent encore à régir, en grande partie, les comportements de certains Africains. Consciemment ou inconsciemment, ils les perpétuent. Parmi ces éléments, hérités du passé, qui demeurent dans nos vies, nous avons les castes. Celles-ci sont perçues par l'étranger, qui se retrouve face à une organisation sociale différente de la sienne, comme une pratique qui bafoue la dignité humaine et empêche le développement économique de certains membres de nos sociétés. Elles sont, de ce point de vue, une absurdité qu'il faut abandonner sans chercher à les comprendre. Pourtant, les castes peuvent nous fournir des informations importantes sur le fonctionnement de notre société et son évolution. C'est pourquoi nous allons, d'abord, essayer de cerner le sens du mot caste ; ensuite, nous tenterons de donner les fondements des castes; enfin, nous examinerons les relations entre les castes.

\section{Caste: un mot au sens équivoque}

Beaucoup de chercheurs, qui se sont intéressés aux castes dans leurs travaux, ont éprouvé la difficulté à donner au mot caste un sens permettant d'en embrasser la quintessence quelle que soit la société étudiée. A titre illustratif, voici la première définition que Louis Dumont, qui a étudié le système des castes en Inde, donne à ce mot :

Le système des castes divise l'ensemble de la société en un grand nombre de groupes héréditaires distingués et reliés par trois caractères: séparation en matière de mariage et de contact direct ou indirect (nourriture); division du travail, chacun de ces groupes ayant une profession traditionnelle ou théorique dont ses membres ne peuvent s'écarter que dans certaines limites; hiérarchie enfin, qui ordonne les groupes en tant que relativement supérieurs et inférieurs les uns aux autres. ${ }^{2}$ (Dumont, 1966: 36)

2 L. Dumont a repris ici la définition de Célestin Bouglé in Essais sur le régime des castes (1908), 1927, p. 4.

Heródoto, Unifesp, Guarulhos, v.4, n.1 - 2019.1. p. 247-263

DOI: $10.34024 /$ herodoto.2019.v4.10121 
Cette acception du mot caste n'est pas pleinement partagée par les chercheurs, comme Abdoulaye Bara Diop qui relève

Les castes peuvent être empiriquement qualifiées sans difficulté; les auteurs sont, dans l'ensemble, d'accord sur leurs principaux caractères apparents, facilement repérables. On les considère, généralement, comme des groupes héréditaires, endogames, à spécialisation professionnelle, entretenant des relations de type hiérarchique. Mais dès qu'il s'agit d'interprétation consistant à définir la notion de caste, à comparer les castes aux autres formes de stratifications sociales, le problème se complique et des divergences apparaissent. ${ }^{3}$ (Diop, 1985: 27)

Partant de l'origine du mot caste et de quelques définitions, nous formulerons notre point de vue sur son acception. Ce mot caste est d'origine latine; il vient de castus qui signifie : chaste, pur. Du latin vient également le mot portugais casta qui veut dire : pur, sans mélange. Est-ce donc à dire que le mot nous renvoie à des faits religieux ou pratiques religieuses, comme le laissent croire ses différentes significations et comme l'estiment certains? Nous pensons que tel n'est pas forcément le cas. La pureté n'est pas seulement l'apanage de la religion. La notion de pureté peut renvoyer à l'état d'un corps qui n'a pas été altéré par un autre. En ce sens donc, le mot latin castus peut bien avoir pour sens : sans mélange, ordonné. C'est ce qui nous amène à considérer, avant tout, la caste comme une organisation bien définie qui ne connaît et $n^{\prime}$ admet en son sein aucun désordre. D'ailleurs, c'est dans cette logique que nous employons le mot caste au sens figuré pour désigner une classe ou un groupe fermé de la société.

Qui plus est, dans le cadre africain, il est extrêmement difficile de faire la distinction entre caste et classe sociale. Nous entendons par classe sociale une autre division de la société, comme l'indique son étymologie latine classis. Caste et classe sociale se superposent et se confondent parfois. Dans la société wolof (ethnie du Sénégal), par exemple, les géer peuvent former aussi bien une caste qu'une classe sociale. Donc les mots peuvent désigner une seule et même chose. C'est ce qu'ont bien compris les rédacteurs du dictionnaire de l'Académie française qui définissent ainsi le mot caste: " chacune des classes de la société dans l'ancienne Egypte, dans l'Inde et dans certains autres pays. ». L'emploi de caste pour désigner les classes sociales en Afrique ou en Inde peut bien être le fait de

\footnotetext{
${ }^{3}$ Dans cet ouvrage consacré aux castes dans la société wolof Abdoulaye B. Diop ne partage pas l'induction de L. Dumont qui part du seul cas hindou pour caractériser toutes les castes. Pour L. Dumont, les castes ont pour fondement la pureté et l'impureté, ce qui n'est pas valable pour toutes les castes à l'image de celles des Wolofs qu'Abdoulaye B. Diop traite dans son œuvre.
}

Heródoto, Unifesp, Guarulhos, v.4, n.1 - 2019.1. p. 247-263

DOI: $10.34024 /$ herodoto.2019.v4.10121 
l'étranger européen qui, face à une organisation sociale différente de la sienne, a voulu la nommer autrement pour illustrer cette particularité.

Ceci est d'autant plus plausible que les Européens ont connu tardivement la caste dont ils limitent souvent l'existence à l'Inde, comme l'atteste la définition du dictionnaire Littré: "Chacune des tribus en lesquelles la société de l'Inde est partagée. Il y a quatre castes: les prêtres, les guerriers, les marchands et agriculteurs, les gens de condition servile, en sanscrit brahmanes, kshatria, vaisia, bûdra.". La remarque qu'apporte ce même dictionnaire est plus révélatrice de cette méconnaissance de la caste en Europe: “caste qui n'est ni dans Furetière ni dans Richelet, n'est dans le dictionnaire de l'Académie qu'à partir de 1740." Au moment où les Européens entraient en contact avec les Africains, cette stratification sociale qu'ils nomment caste avait déjà fait sa mue au point qu'il est difficile de retracer son origine et son évolution. Toutefois, une lecture des auteurs anciens nous conforte dans l'idée que les castes, comme nous les appelons de nos jours, ne sont que le fruit d'une simple organisation de la société en différentes classes productrices. Hérodote l'indique clairement:

Il y a sept classes d'Egyptiens, qu'on appelle les prêtres, les guerriers, les bouviers, les porchers, les marchands, les interprètes, les pilotes. Tel est le nombre des classes en Egypte, et leurs dénominations viennent des professions exercées. (Hérodote, 1989: 164)

Nous considérons donc que les castes sont une stratification sociale, à l'image de celles connues en Europe. Elles sont établies sur la base des activités professionnelles, des métiers et nous partirons des textes anciens pour mieux percevoir les fondements et les évolutions.

\section{Les fondements des castes}

\section{a- Le fondement socio - politique}

Les sociétés africaines, à l'instar de toutes les autres, ont connu dans le passé des formes d'organisation particulières. D'une part, l'organisation sociale se manifeste au sein même de la famille où chaque membre avait un rôle bien déterminé à remplir pour le bien- être de tous. Les tâches étaient dévolues en fonction de l'âge, de la capacité physique, morale, mais également du sexe. D'autre part, dans un cadre un peu élargi, celui de la tribu ou de l'ethnie, on retrouve également des distributions de rôles. La seule différence étant qu'un membre d'une famille peut jouer un autre rôle au sein du clan, de la tribu ou de l'ethnie. Ceci s'explique par le 
fait que l'aîné de la famille n'est pas obligatoirement celui de la tribu. Chacune de ces deux entités a à sa tête un chef qui veille à l'exécution par chacun du travail qui lui est assigné. C'est cette forme d'organisation qui déterminait également les rapports entre les différents membres de la famille ou de la tribu.

Par ailleurs, c'est cette organisation sociale qui a favorisé le développement de l'économie agricole en Afrique ancienne. En effet, en cette période où les champs étaient communautaires, il fallait bien une bonne organisation dans le travail pour espérer avoir une bonne récolte. Ainsi à chaque période des cultures, il y eut des gens préposés aux débroussailles, d'autres aux tracés des sillons, d'autres à l'enfouissement des graines dans le sol. Durant le temps des travaux, tout se déroulait en synergie, de la préparation des champs aux semailles, en passant par l'arrachage des mauvaises herbes, la moisson, jusqu'à l'acheminement des récoltes dans les foyers. Force est, d'ailleurs, de constater que cette organisation sociale est antérieure au début de l'agriculture. Elle tire son origine lointaine de la période de la chasse et de la cueillette. A ce moment là, la chasse à l'épieu ou à l'arc était réservée aux plus endurants à la course et aux plus habiles aux tirs. Les autres membres de la tribu ou du clan se répartissaient le transport des bagages (armes, gibiers...), la préparation du feu, la cuisson et autres charges.

Cette organisation sociale, dans le travail, va se consolider et s'améliorer dès l'apparition des premières structures étatiques. Les Africains, comme tous les autres peuples, vivaient en communautés dont l'exigence d'une harmonie sociale imposa une organisation politique. Les hommes sentirent la nécessité de mettre à leur tête quelqu'un pour maintenir l'ordre social, pour assurer la prospérité et la défense communautaires. C'est ainsi qu'ils élurent des dirigeants appelés rois et l'ensemble de toute la communauté constitua le royaume. Au sujet du choix du roi, voici ce qu'écrivit Diodore de Sicile:

Quant à la royauté, les uns la remettent entre les mains des hommes les plus beaux, estimant que beauté et pouvoir monarchique sont tous deux des dons du sort, les autres confient le pouvoir à ceux qui soignent le mieux leurs troupeaux, dans la pensée que, seuls, ils prendront parfaitement soin de leurs sujets; quelques-uns attribuent cet honneur aux plus riches, estimant que seuls ceux-ci peuvent protéger le peuple à cause des facilités que leur donne leur fortune ; il y en a enfin qui choisissent pour rois ceux qui se font remarquer par leur courage,

Heródoto, Unifesp, Guarulhos, v.4, n.1 - 2019.1. p. 247-263

DOI: 10.34024/herodoto.2019.v4.10121 
jugeant que les plus capables à la guerre sont seuls dignes d'obtenir le premier rang. ${ }^{4}$ (Sicile, 1989: 4)

Le roi gouvernait, assisté par un conseil des sages. Au début, son pouvoir n'était pas absolu et ses décisions étaient orientées dans le sens de l'intérêt commun. Lorsque survint le passage d'une économie agricole vers une économie monétaire, dictée par les échanges commerciaux avec l'étranger, le roi d'Egypte dut prendre cette décision que nous révèle Hérodote:

Il y a sept classes d'Egyptiens, qu'on appelle les prêtres, les guerriers, les bouviers, les porchers, les marchands, les interprètes, les pilotes. Tel est le nombre des classes en Egypte, et leurs dénominations viennent des professions exercées. Ceux d'entre eux qui forment la classe des guerriers sont appelés Calasiries et Hermotybies... A eux non plus il n'est permis d'exercer aucun métier, sinon le métier de la guerre, où ils se succèdent de père en fils. ${ }^{5}$ (Hérodote, 1989: 164, 166)

Cette division de l'Egypte en différentes classes, dont chacune renvoie à un métier précis, semble être une consolidation et une adaptation de la division du travail, connue depuis l'étape clanique, face aux mutations historiques. Les échanges commerciaux avec le monde grec, par exemple, étaient très développés et concernaient des produits divers. Il fallait produire différents articles pour assurer la prospérité économique de l'Egypte et garantir en même temps la sécurité interne et externe du royaume. Pour ce faire, une professionnalisation des Egyptiens était nécessaire; ce que comprirent les pharaons qui procédèrent à cette répartition des tâches. Ainsi, chaque corps de métier se perfectionnait dans son domaine et avait une totale sérénité pour exécuter son travail. A titre illustratif, le marchand pouvait commercer à longueur de journée sans se soucier de l'entretien de ses bêtes.

Bref, les informations qui nous sont fournies par Hérodote sont très précieuses pour comprendre les castes. Elles nous apprennent, d'une part, que ce que nous appelons aujourd'hui les castes en Afrique est une division du travail consolidée et améliorée par une décision politique qui va semer les germes de la professionnalisation et de l'hérédité du métier. D'autre part, elles nous renseignent qu'il n'y avait pas, au début, de

\footnotetext{
${ }^{4}$ L'élection du roi est une manifestation de l'égalité de tous les membres de la communauté en dépit des fonctions exercées par les uns et les autres. Aucun critère discriminatoire n'était établi pour écarter un membre de telle ou telle autre fonction.

${ }^{5}$ Les pilotes étaient des marins qui gouvernaient les bateaux marchands, les Calasiries seraient des soldats de l'infanterie mercenaire nubienne et les Hermotybies des conducteurs de char.
}

Heródoto, Unifesp, Guarulhos, v.4, n.1 - 2019.1. p. 247-263

DOI: $10.34024 /$ herodoto.2019.v4.10121 
critères de supériorité ou d'infériorité définis entre gens de différentes professions. Ceci est d'ailleurs confirmé par ces propos:

Il y eut à l'origine une division du travail social avec la séparation des métiers de l'agriculture sans aucune idée de hiérarchie. C'est le phénomène tel qu'on le voit encore, de nos jours, dans les pays de forêt du sud (Casamance, Baoulé, etc.) (Diop, 1985: 43)

Cependant, Hérodote a passé sous silence des corps de métiers importants en Egypte ancienne, comme celui des cultivateurs. Cette omission nous conduit à visiter les écrits de Diodore de Sicile qui, des années après Hérodote, note ceci à propos des classes égyptiennes:

Le commun des habitants est divisé en trois classes : les laboureurs, les pasteurs et les artisans. Les laboureurs prennent pour un temps, à un prix modique, les terres du roi ou des prêtres ou des soldats et emploient tout ce temps à les cultiver. Étant nés dans ces exercices ils savent mieux l'agriculture qu'on ne la sait partout ailleurs. Ils connaissent parfaitement la nature des terres, les temps des débordements du Nil, la saison propre aux semailles, aux moissons et aux transports des denrées soit par les instructions qu'ils ont reçues de leurs pères, soit par les épreuves qu'ils ont faites eux-mêmes. Il en est ainsi des pasteurs qui ont reçu de leurs parents comme par héritage la connaissance de tout ce qui regarde les troupeaux, qui l'ont cultivée par une longue habitude et qui de plus inventent souvent des manières nouvelles d'augmenter les profits qu'on peut tirer des bestiaux. Ce qu'il y a de plus particulier est que ceux qui élèvent des oiseaux de basse-cour, trouvent par leur application et par leur industrie des moyens de les faire multiplier tout autres que les voies ordinaires qu'il semble que la nature ait établies pour cet effet. Car au lieu de laisser couver les œufs par les oiseaux mêmes qui les ont pondus, ils ont la patience de les faire éclore en les échauffant dans leurs mains. Par là ils avancent l'ouvrage de la nature et ils augmentent considérablement ses productions. Mais rien n'est plus admirable que l'utilité et la perfection des arts qui s'exercent chez les Égyptiens. C'est le seul pays du monde où ceux qui sont nés dans une profession et qui pour ainsi dire l'ont reçue des lois, ne la quittent jamais pour en exercer une autre, de sorte que ni les jalousies domestiques, ni leur ambition particulière, ne les tirent jamais de la profession paternelle. ${ }^{6}$ (Sicile, 1737: XXV)

Les propos de Diodore confirment non seulement le critère d'hérédité dans la transmission des métiers, mais aussi nous apprennent que les professions n'étaient pas attribuées au hasard. A chacun était assigné, à l'origine, le métier où il avait une prédisposition naturelle. Au

\footnotetext{
6 Mises en parallèle, les trois classes de Diodore et les sept classes d'Hérodote nous révèlent qu'une classe pouvait comporter plusieurs métiers ou se subdiviser en sous classes en fonction de la différence de profession. En effet, les bouviers et les porchers relevés par Hérodote forment la classe des pasteurs chez Diodore. De même, la classe des artisans dont parle Diodore va donner naissance à d'autres sous-classes, comme celle des barbiers, selon Babacar Diop dit Buuba dans son livre Propos d'un Africain sur l'Antiquité, p.114.
}

Heródoto, Unifesp, Guarulhos, v.4, n.1 - 2019.1. p. 247-263

DOI: $10.34024 /$ herodoto.2019.v4.10121 
demeurant, les classes sociales, qui seront appelées des castes par la postérité, sont bien issues d'une division du travail qui a un fondement socio - politique. Elles se maintiendront dans beaucoup de sociétés africaines surtout celles d'Afrique de l'ouest où elles existent chez les Wolofs, les Peulhs, les Sénoufo, les Maures...

Le fondement socio- politique des castes s'est manifesté lors de la naissance de la caste des griots qui serait originaire du Mali. Les griots forment la grande caste de ceux qui doivent vivre de leur parole. Parmi eux nous avons les musiciens, les chanteurs et les laudateurs. La caste est créée par le roi de l'empire du Mali Soundjata Keita après sa victoire sur le roi du Sosso, Soumangourou Kanté. Selon Tal Tamari, repris par Gilles Holder (Holder, 1999: 236), Sounjata avait attribué aux leaders sosso la fonction de conseillers, puis d'intermédiaires ensuite de maîtres de cérémonie et d'envoyés. Ceux-ci formèrent ainsi la caste des griots.

En somme, nous pouvons clore ce premier point en retenant que le premier fondement des castes est socio- politique; ce que partage pleinement le savant sénégalais Cheikh Anta Diop qui écrivit:

Le système est né d'une division du travail mais sous un régime politique avancé, monarchique, car on ne trouve jamais de castes sans noblesse. Cependant, il est fort probable que la spécialisation dans le travail, qui a abouti à l'hérédité du métier dans le système des castes à l'échelle familiale et individuelle, s'est élaborée depuis l'organisation clanique. (Diop, 1960: 11)

Toutefois, ce seul critère professionnel ne peut expliquer aujourd'hui les autres caractéristiques des castes, à savoir l'hérédité, l'endogamie et la hiérarchie. Pour mieux comprendre les castes, nous sommes obligé de partir de leur état présent pour voir comment d'autres facteurs, comme celui religieux, ont contribué, dans le passé et même encore de nos jours, à leur maintien et à leur légitimation.

\section{b- Le fondement religieux}

Les castes sont issues de la division du travail au sein de la société depuis l'organisation clanique. Elles ont été raffinées en Egypte ancienne par les pharaons. Ceux- ci avaient réparti la société en différentes classes formant des corps de métier et ils veillaient strictement au maintien de celles-ci. La mesure phare prise, à l'époque, par les pharaons pour faire respecter et perpétuer cette division sociale favorisa l'instauration du critère d'hérédité. Désormais, le métier était héréditaire. Pour éviter que des ambitieux ou des jaloux ne changeassent de métier, suivant leur bon 
vouloir, des sanctions furent prévues par les lois égyptiennes. Nous y reviendrons. Ce fut donc, au début, la crainte de l'autorité qui favorisa la survie des classes professionnelles, qui se mueront en castes, en Egypte ancienne. Ceci est très compréhensible lorsqu'on sait que le pharaon avait droit de vie et de mort sur ses sujets.

Mais, nous estimons qu'une simple rationalité humaine ne suffit pas à perpétuer, durant des siècles, une telle stratification sociale qui a eu à faire, à coup sûr, beaucoup de mécontents. En effet, il y eut bien en Egypte ancienne des gens dédaigneux du métier dont ils avaient hérité et qui voulurent changer de classe ou de catégorie sociale à tout prix. A ceux-ci, il fallait plus qu'une sanction pécuniaire ou quelques coups de fouet pour freiner les ardeurs. Il leur était nécessaire un mythe, un mystère qui inspira une telle peur ou une telle horreur à vivre pour qu'ils ne songeassent jamais à changer de classe ou à accueillir un autre dans la leur. Un fondement pareil ne pouvait provenir que de la religion. A un moment donné de l'évolution des classes, les hommes ont dû faire appel à la religion, et ce peut être depuis l'Egypte pharaonique, pour légitimer et assurer la survie de cette répartition sociale à base professionnelle. La religion avait un immense pouvoir en Egypte. Elle rythmait la vie des Egyptiens et déterminait leur conduite. Le sacré était vénéré et nul n'osait enfreindre les lois divines. Il était alors l'idéal pour consolider, voire donner un autre fondement aux castes. La religion n'a-t- elle pas, d'ailleurs, beaucoup aidé à assoir le pouvoir du pharaon ? Celui-ci était considéré comme le fils du dieu Soleil, Râ. C'est pourquoi il était le maître de l'univers pour les Egyptiens ; ce qui explique son droit de vie et de mort sur tout être vivant.

C'est la religion qui consacra la hiérarchie entre les classes basée sur la notion de pur et d'impur qui favorisa l'endogamie. Partie d'un simple désir de maintenir l'existence des classes, elle scellera définitivement leur fixité, leur fermeté et leur hiérarchie. C'est pour cette raison qu'elle apparait, aux yeux de beaucoup de modernes, comme le fondement principal des castes. C'est ce qui justifie pleinement cette appellation de castes, à partir du XVIIème siècle, à ce qui n'était avant que de simples classes professionnelles. Ceci n'a pas échappé à l'œil perçant de L. Dumont (Dumont, 1966: 103) qui, tout en partant du système varna dans son étude des castes en Inde, fonde les castes sur l'opposition entre le pur et l'impur.

Mieux encore, cette notion de pur et d'impur, apportée comme critère dans les castes par la religion, a un double impact sur le système. D'une part, elle s'applique au métier. La profession exercée peut être considérée 
comme pure ou impure ${ }^{7}$ par les membres d'une autre classe. Les métiers de l'artisanat sont jugés impurs et donc indignes pour ceux qui appartiennent aux castes supérieures, comme celles des intellectuels, des guerriers et des prêtres. Ils sont ainsi considérés à cause de leurs effets dégradants sur le corps humain ou à cause des produits travaillés. C'est ainsi que la sous-caste wolof des cordonniers est inconsidérée, voire méprisée par les géer, à cause des peaux en putréfaction qu'elle manipule. L'odeur pestilentielle, dans laquelle baignent les cordonniers, souille leur être et pousse les autres à les tenir à l'écart. En somme, ce sont tous les métiers manuels, à l'exception de l'agriculture, qui sont qualifiés d'impur dans les sociétés où existent des castes.

D'autre part, dans le système des castes, la pureté ou l'impureté peut être liée à un jugement racial que conforte la religion traditionnelle. Dans toutes les sociétés africaines, ceux qui appartiennent aux castes supérieures, formées pour la plupart d' intellectuels, de religieux, de guerriers et de cultivateurs, croient en leur supériorité raciale sur les autres. Ils s'estiment issus d'un sang pur qui n'a pas été souillé. Ils attribuent aux autres des origines peu glorieuses qui entraîneraient la malédiction de quiconque s'unirait à eux. Ce rejet des castes inférieures, dans les croyances populaires, a une justification divine. Par exemple, chez les guélwar sereer (ethnie du Sénégal), certains attribuent l'infériorité raciale des griots à leur origine servile, d'autres à une punition divine de leur ancêtre déchu pour inceste. Une telle pensée, ou croyance, que l'on justifie par la volonté des dieux explique les traitements qui étaient réservés aux griots en milieu sereer, comme l'illustre ce passage:

Leurs corps empoisonneraient les grains et les fruits, prétendent les autres nègres, et ils empoisonneraient également l'eau et les poissons ; alors ils ne sont ni enterrés ni jetés dans la mer ou les rivières. Les griots passent, en outre, pour vivre dans un commerce familier avec le diable, et cette croyance ne contribue pas peu à leur interdire l'accès des lieux de sépulture ordinaire. ${ }^{8}$ (Raffenel, 1846: 19)

Pour le guélwar sereer, comme pour les autres membres des castes supérieurs en Afrique, l'union avec un homme ou une femme d'une caste inférieure ne peut demeurer impunie des dieux. L'auteur subit toujours un malheur physique ou économique. Il est aussi méprisé par ses proches parents et même par les membres des autres castes qui jugent son sang souillé. Ainsi, la religion traditionnelle a œuvré au maintien des castes et

\footnotetext{
${ }^{7}$ Pur et impur doivent être compris ici dans le sens de noble et de roturier.

${ }^{8}$ La sépulture des griots était les troncs creux des baobabs où l'on suspendait leur corps sur des grabats avant de refermer l'ouverture qui n'était réouverte que pour un nouveau corps.
}

Heródoto, Unifesp, Guarulhos, v.4, n.1 - 2019.1. p. 247-263

DOI: $10.34024 /$ herodoto.2019.v4.10121 
favorisé l'endogamie. Comme le fondement socio- politique, elle a joué un rôle très important dans le système des castes. Comment se fait donc la balance? Quelle est la part de l'idéologie et celle de l'institution judiciaire?

\section{c- Le fondement juridique}

La législation des sociétés africaines, au cours de l'Antiquité, est mal connue. Les lois qui régissaient les rapports au sein de la société n'étaient pas toujours écrites. Elles étaient orales dans la plupart des groupes sociaux. C'est pourquoi beaucoup n'ont pas survécu au temps. Elles ont subi des modifications au cours de leur transmission ou sombré dans l'oubli total face aux bouleversements sociaux nés du contact avec l'étranger. En Egypte ancienne, où les hommes ont laissé beaucoup de traces écrites, quelques lois nous sont parvenues grâce aux auteurs grecs, comme Hérodote et Diodore de Sicile. D'ailleurs, c'est ce dernier qui nous apprend que la loi égyptienne a participé à la formation et à la conservation des castes. A ce sujet, Diodore écrivit:

On voit fort souvent chez les autres peuples que les jeunes gens par légèreté d'esprit ou par envie de gagner davantage, se dégoûtent de la profession de leurs parents ou s'appliquent à diverses choses à la fois... Mais chez les Égyptiens si quelque artisan se mêlait des affaires d'état, quittait sa profession pour en prendre une autre ou en voulait exercer plus d'une, il était grièvement puni. ${ }^{9}$

Il est donc clair que la loi a servi, dans le passé, de fondement à la naissance des classes sociales devenues, plus tard, des castes. Il ne pouvait en être qu'ainsi puisque, dans bien des sociétés anciennes, la décision du roi devenait souvent une loi. Le roi avait un pouvoir absolu qui faisait de lui un législateur. Dans ce cas précis, il aurait été intéressant de connaître l'exposé des motifs de cette loi égyptienne relative aux classes mais Diodore ne l'indique pas. Toutefois, la sanction infligée à celui qui abandonnait sa classe est contenue dans ces propos: "Quatrièmement, il était enjoint à tous les Égyptiens de déclarer leur nom, leur profession et leurs revenus aux magistrats, et l'on condamnait à la mort celui qui faisait une fausse déclaration ou qui exerçait un métier illicite." 10 La sévérité de cette peine fit de la loi une actrice majeure dans l'élaboration et la survie des castes qu'elle avait ainsi légitimées. Nous

\footnotetext{
${ }^{9}$. Diodore de Sicile, Bibliothèque historique I, section seconde, XXV.

${ }^{10}$. Id. ; ibid. ; XXVII. La peine capitale infligée ici au contrevenant contraste bien avec ce que dit Diodore dans la première citation «Il était grièvement puni ». Cependant, nous pensons que c'était bien la peine de mort qui était appliquée pour éviter l'écroulement du système des castes.
}

Heródoto, Unifesp, Guarulhos, v.4, n.1 - 2019.1. p. 247-263

DOI: $10.34024 /$ herodoto.2019.v4.10121 
pouvons alors retenir que la justice, au même titre que la politique et la religion, a été un élément de base des castes en Afrique. Ces trois principes nous fournissent de précieuses informations sur celles-ci, mais ils ne nous permettent pas de comprendre leur fonctionnement. C'est pourquoi, nous allons, dans le point suivant de notre travail, nous intéresser aux relations entre les castes.

\section{Les relations entre les castes}

Les castes africaines sont nées, comme toutes les autres en réalité, de la division du travail. Leur origine laisse déjà présager des relations intenses au sein de la société. S'étant tournés vers l'agriculture, les hommes se rendirent vite compte de la nécessité de se répartir les tâches; ce qui conduisit progressivement à une spécialisation. Celle-ci sera accentuée par les échanges tant internes qu'externes. Il y avait une interdépendance des différentes classes sociales ou castes. Aucune ne pouvait vivre sans le concours de l'autre. Ce fut une complémentarité qui rendait chacune indispensable au bien-être de la communauté. En examinant les différentes classes de l'Egypte ancienne, dont nous ont parlé Hérodote et Diodore de Sicile, on s'en rend vite compte. Avec Hérodote, nous découvrons que les prêtres s'occupaient de l'éducation morale en enseignant aux citoyens les principes religieux fondés sur la connaissance $\mathrm{du}$ bien et du mal; les intellectuels transmettaient les connaissances scientifiques ; les guerriers assuraient la sécurité de tous et la gestion de la cité, car, parmi eux, était le roi et ses services ; les marchands favorisaient la prospérité économique. Le rôle de chacune de ces classes est donc important pour la cité. Il suffit de supprimer l'une d'entre elles pour que le chaos s'installe. Cette complémentarité est plus apparente dans la relation qui unit les artisans, les cultivateurs et les guerriers. Sans les premiers qui fabriquent les instruments aratoires et les armes, les seconds sont dans le désarroi. Non plus, les artisans ne peuvent exister sans les paysans et les soldats qui leur procurent vivres et sécurité.

Pour garantir l'ordre et l'équilibre social, une quantité déterminée de productions était assignée aux paysans et aux artisans. Un contrôleur des productions s'assurait que ceux-ci produisirent plus pour favoriser la prospérité économique du pays. Le peuple tout entier avait une grande estime pour les artisans dont l'art et l'amour du métier émerveillaient plus d'un. C'est pourquoi les pharaons veillaient à ce que les artisans ne manquassent de rien, comme l'illustre ce passage:

Heródoto, Unifesp, Guarulhos, v.4, n.1 - 2019.1. p. 247-263

DOI: $10.34024 /$ herodoto.2019.v4.10121 
Pour vous, les greniers seront gonflés de blé...; chacun d'entre vous aura des provisions pour un mois. J'ai aussi rempli les magasins de toutes sortes de choses, pains, viandes, gâteaux pour vous protéger (de la faim), des sandales, des vêtements, de nombreux onguents... J'ai aussi mis en place un nombreux personnel pour subvenir à vos besoins: des pêcheurs vous apporteront des poissons, d'autres, des jardiniers, feront pousser des légumes; des potiers travailleront au four afin de fabriquer de nombreuses cruches, ainsi, pour vous, l'eau sera fraîche à la saison d'été...11 (Obenga, 1990: 452)

Bref, les castes étaient fondées sur un échange de services et de biens et tous leurs membres étaient d'égale dignité. Chaque membre d'une caste était conscient des liens avec d'autres et en tenait compte. Cependant, avec l'avènement de la monarchie et les contacts extérieurs, l'inégalité va s'installer progressivement entre les castes. On va assister à une hiérarchisation de celles-ci. Cette hiérarchie des castes doit être comprise dans ce sens que lui donne L. Dumont: "Principe de gradation des éléments d'un ensemble par référence à l'ensemble." (Dumont, 1966: 92)

La hiérarchie des castes africaines a une double origine. Elle provient d'abord de la confiscation du pouvoir par les détenteurs d'autorités dont sont issus les rois. Ceux-ci vont s'imposer à tous par la force et considérer les autres comme leurs sujets. Conscients du rôle que jouent la religion et l'éducation dans la société, ils vont attribuer aux intellectuels la deuxième place dans la hiérarchie.

En effet, même si les pharaons ont été très conscients du pouvoir des prêtres dans la société, ils n'ignorèrent pas pour autant celui des hommes de lettres, de sciences et de techniques. Ils savaient très bien l'importance de l'éducation qui était la clé de la grandeur morale de l'homme et le socle $\mathrm{du}$ développement économique d'un pays. C'est la raison pour laquelle ils mirent les intellectuels au premier rang de la société pour baliser les voies du développement et guider le peuple vers le droit chemin. Les scribes occupaient d'éminentes fonctions dans l'administration, avaient de nombreux avantages et étaient admirés par tous. Chacun voulait avoir un fils scribe car cette profession fut la plus prestigieuse au bord du Nil.

Les autres castes se succèderont en fonction de l'importance de leur métier pour le pouvoir central. Ainsi les prêtres et les soldats étaient parmi les privilégiés de la société égyptienne, comme l'a souligné Hérodote: “A l'exclusion de tous les Egyptiens, sauf des prêtres, les gens

\footnotetext{
${ }^{11}$ Ces propos de Ramsès II, extraits d'une inscription sculptée sur une stèle découverte à Manshiyet es- Sadr et datant de l'an 8 de son règne, montrent non seulement toute la gratuite du pharaon pour les ouvriers et les artisans mais aussi la considération qu'avait le peuple pour ces derniers.
}

Heródoto, Unifesp, Guarulhos, v.4, n.1 - 2019.1. p. 247-263

DOI: $10.34024 /$ herodoto.2019.v4.10121 
de guerre avaient des avantages spéciaux, que voici : à chacun étaient attribuées douze aroures de terre, franches d'impôts." 12 (Heródoto, 1989: 168)

Dans cette hiérarchie des castes, la dernière place est, presque dans toutes les sociétés africaines, occupée par les artisans à cause de leur métier qui exige des efforts physiques pénibles et la manipulation de produits incommodants ou salissants. Ce dédain des professions manuelles, sauf l'agriculture, est ainsi manifesté dans l'enseignement de Khéti :

J'ai vu le métallurgiste au travail, à la gueule de sa fournaise. Ses doigts sont comme la peau d'un crocodile, il sent plus mauvais que le frai de poisson. Le menuisier qui manie l'herminette, il est plus harassé encore que le paysan. Son champ à lui c'est le bois, et sa houe c'est le foret. Au soir ; il est exténué, car il a travaillé au-delà de ses forces et pourtant, la nuit, il y a encore de la lumière chez lui. Le tailleur de pierres grave au ciseau toutes sortes de pierres dures. Lorsqu'il a terminé son travail, ses bras l'abandonnent, il n'en peut plus. Lorsqu'à la tombée du jour il s'assied, ses genoux et son dos sont brisés. Le potier baigne dans son argile. Ayant cuit ses vases, il est plus crotté qu'un porc. Ses vêtements sont raides d'argile. L'air brûlant qui sort du four lui passe sur le visage. Il gâche l'argile avec ses pieds, et se brise à cette tâche. Le maçon qui bâtit, il est toujours exposé au vent. Il construit, vêtu d'un simple pagne et ses bras baignent dans l'argile dont tous ses vêtements sont maculés. Il doit manger son pain avec des doigts mal propres. Le tisserand vit dans son atelier. Il est plus mal à l'aise qu'une famille qui accouche. Les genoux repliés contre l'estomac, il suffoque. Passe-t-il un jour sans tisser, on le bat de cinquante coups de nerf, et il doit donner un pourboire à son potier pour pouvoir sortir à l'air. Le cordonnier, dont le sort est bien misérable, porte perpétuellement ses cuves à tanner. Il n'en mène pas plus large que celui qui vit parmi les cadavres. Tout ce qu'il mange a un goût de cuir. ${ }^{13}$ (Obenga, 1846: 487)

Ensuite, l'autre source de la hiérarchie est raciale. L'idéologie d'une race pure qui ne doit pas se mêler aux autres a alimenté un fort sentiment de supériorité dans le système des castes. Ceux qui détiennent la force considèrent toujours les autres issus d'une naissance inférieure, frappés d'une malédiction ou condamnés à une servitude. Cette considération raciale, qui justifie la hiérarchie des castes, explique l'endogamie. A. B.

${ }^{12}$ Les intellectuels étaient parmi ces privilégiés même si Hérodote ne les a pas mentionnés avec leurs avantages. Les scribes étaient les premiers collaborateurs des pharaons; ils bénéficiaient d'une délégation de pouvoir et de nombreuses largesses royales.

${ }^{13}$ Ce long passage, qui date de la XIIème dynastie égyptienne, est un exposé d'un père, Khéti, qui cherche à montrer son fils Pépi, qu'il amène à son premier jour de classe, le mépris qu'ont les Egyptiens pour les métiers manuels et à ressortir l'importance des études qui apparaissent comme l'unique source d'épanouissement physique et social. Hérodote a également souligné ce mépris des professions manuelles dans Histoires II, 164-166.

Heródoto, Unifesp, Guarulhos, v.4, n.1 - 2019.1. p. 247-263

DOI: $10.34024 /$ herodoto.2019.v4.10121 
Diop le souligne bien en ces termes: "L'endogamie est la conséquence logique directe de l'idéologie de castes, dont nous avons noté le caractère biologique, faisant de celles-ci comme des races différentes hiérarchisées." (Diop, 1981: 65)

Enfin, la hiérarchisation des castes a entraîné le mépris des métiers et des hommes. Les castes supérieures jugent les professions manuelles, excepté l'agriculture, indignes d'un homme libre et évitent ceux qui les pratiquent, les considérant comme des parias. Les membres des castes inférieures se marient entre eux et leur accès à certains endroits est limité. Ils sont accusés de tous les maux et écartés de la gestion du pouvoir.

\section{Conclusion}

Les castes résultent d'une division de la société en différentes classes ou catégories sociales. Elles ont eu d'abord un fondement socio- politique qui leur a permis de se maintenir et de s'adapter aux exigences du temps. $C^{\prime}$ est dans le souci de les pérenniser que les hommes vont ensuite leur donner des fondements religieux et juridique. La religion, surtout, va consolider les castes en ajoutant à leur caractère héréditaire l'endogamie et la hiérarchie. L'importance de ces deux derniers facteurs dans le système des castes fait souvent croire aux chercheurs qu'elles ont une origine religieuse. $S^{\prime} y$ ajoute que les rapports entre les castes sont dictés par des considérations religieuses. Le mépris et la restriction des contacts entre gens de castes différentes sont alimentés par des idéologies raciales que justifie la religion. Ceci est encore observable dans plusieurs sociétés africaines où subsistent les castes.

Cependant, il est important de noter, aujourd'hui, que le système des castes a tendance à disparaître. L'économie monétaire a supprimé la dépendance entre les castes. Dans beaucoup de sociétés, le pouvoir financier, et même parfois politique, est détenu par des gens de castes dites jadis inférieures. Les idéologies d'égalité de naissance et de droit prônées par le Christianisme, l'Islam, et défendues par nos juridictions modernes, combattent toute velléité de maintenir les castes vues comme une aberration, une aliénation. Ainsi un retour aux sources anciennes est nécessaire pour comprendre cet héritage qui vivote parmi nous. 


\section{Auteurs anciens}

DIODORE DE SICILE. Bibliothèque historique II, texte établi et traduit par B. Bommelaer, Paris, Les Belles Lettres, 1989.

DIODORE DE SICILE. Bibliothèque historique III, texte établi et traduit par B. Bommelaer, Paris, Les Belles Lettres, 1989.

HÉRODOTE. Histoires II, texte établi et traduit par Ph. E. Legrand, Paris, Les Belles Lettres, 1989.

\section{Auteurs modernes}

DIOP, Abdoulaye Bara. La société wolof, tradition et changement, les systèmes d'inégalité et de domination, éditions Karthala, 19

DIOP, Babacar dit Buuba. Propos d'un Africain sur l'Antiquité, Dakar, Presses Universitaires de Dakar, 2014.

DIOP, Cheikh Anta. L'Afrique noire précoloniale, Paris, Présence Africaine, 1960.

DIOP, Majhemout. Histoire des classes sociales dans l'Afrique de l'ouest, tome I : Le Mali, Paris, éditions l’Harmattan, 1985.

DUMONT, Louis. Homo hierarchicus, Essai sur le système des castes, Paris, Gallimard, 1966

HOLDER, Gilles. Tal Tamari, Les castes de l'Afrique occidentale. Artisans et musiciens endogames. In : «L'Homme », tome 39 n¹52, 1999.

OBENGA, Théophile. La philosophie africaine de la période pharaonique 2780330 avant notre ère, Paris, L'Harmattan, 1990.

RAFFENEL, Anne. Voyage dans l'Afrique occidentale en 1843 et 1844, Paris, Bestrade, 1846. 